\title{
Unusual Suspect of Societal Innovativeness in Online Social Innovation Community: A Network and Communication Framework
}

\author{
Jemin Justin Lee ${ }^{1}$, Youngjoon Cheon ${ }^{2}$, Sangyun Han $^{3}$ and Kyu Tae Kwak ${ }^{4 *}$ \\ ${ }^{1}$ Department of Technology and Business Administration, Yonsei University \\ Seoul, South Korea \\ [e-mail: justin.lee@yonsei.ac.kr] \\ ${ }^{2}$ Youngpoong corporation \\ Seoul, South Korea \\ [e-mail: taisama@naver.com] \\ ${ }^{3}$ Department of International Commerce, Daejeon University \\ Daejeon, South Korea \\ [e-mail: syhan@dju.ac.kr] \\ ${ }^{4}$ Department of Global Culture Industry, Soonchunhyang University \\ Asan, Chungcheongnam-do, South Korea \\ [e-mail: ktkwak@sch.ac.kr] \\ *Corresponding author: Kyu Tae Kwak
}

Received November 19, 2017; revised May 20, 2018; accepted July 16, 2018;

published December 31, 2018

\begin{abstract}
The widespread adoption of the social computing paradigm has ushered in the development of online social innovation community (OSIC) as a promising method for solving social problems. Previous studies have not explicitly considered the conceptual factors that facilitate these communities' users' innovative activities, so it is vital to conduct empirical studies to verify the effectiveness of these factors. In this paper, the primary goals are to construct a theoretical model of the social innovation and empirically verify the casual relationship between theoretical factors and societal innovativeness. A survey of 398 OSIC users was conducted to empirically validate the theoretical model. The causal relationships between network characteristics and social innovativeness were experimentally tested. The results of this study indicated that ambiguity, switching, and multiplexity are important factors that facilitate social innovativeness, which contradicts the prior assumptions about innovation performance.
\end{abstract}

Keywords: Societal innovativeness, social innovation, OSIC, network, experimentation

A preliminary version of this paper was presented at APIC-IST 2016 and this version includes a concrete analysis and enhances theoretical discussion. And also this work was supported by the Soonchunhyang University Research Fund (No.20170267). 


\section{Introduction}

In recent years, solving societal problems through online innovation communities has received a significant amount of attention in various fields [1]. A notable example of the online social innovation community is Ushahidi, an open-source mapping technology created during the natural disaster in Haiti, which utilized geographic information to help solve the human rights problems with the help of volunteers participating through the online community [2]. Recent studies regarding open innovation and co-creation have asserted that the creativity and innovativeness to solve the social challenges can be developed when members of the online communities share their opinions with one another [2][3]. Online communities have successfully facilitated knowledge transfer and encouraged voluntary participation to solve many societal problems. IDEO's Design Kit platform has emerged as a plausible framework for online service design community that can help generate user's knowledge and develop alternative technical solutions to resolve societal problems. Design Kit participants not only support developing countries by sharing their insight and expertise about digital solutions but will also contribute to the implementation of various physical solutions as well. These platforms, where people can discuss and resolve social problems, are the online social innovation community (hereinafter "OSIC").

Related studies pertaining to the OSIC platform have focused on micro-volunteering and help-giving behaviors in the online environment [4][5]. Most of these studies focused on practical methods for developing technical systems or provided qualitative descriptions of a few successful examples [5], but they did not investigate the foundation of collaborative and pro-social behaviors in OSIC. In particular, past studies have not investigated the interactive problem-solving process and relational environment that affect the interpersonal communications in the OSIC.

In addition, most studies on the OSICs are intrinsically limited to the degree to which their results can be generalized, from socio-economic constructs to pro-social behaviors in online environment. Few studies have investigated societal problem-solving activities from the perspective of traditional constructs in peer pressure and the indivdual's motivation [6]. However, previous studies have not investigated the complex characteristics of OSCI, such as fluidity, goal ambiguity, and dispersion of attention [7]. Due to the lack of empirical and theoretical evidence about these factors, it is not clear what conditions would influence the user's ideation and voluntary activities in online social innovation platform.

The research goal of this study is to investigate which factors were critical for facilitating social innovativeness, which is defined as a method that online users propose innovative ideas for solving social problems. We focus on interactive problem solving factors closely related to the OSIC's characteristics. In order to achieve the research goal, we considered ambiguity, switching, multiplexity, and cohesiveness as critical factors from the network and communication theoretical framework [8][9][10]. White et al. [10] developed this framework to explain the complex features of the contemporary societies, while focusing on the role that linguistics and stories played in various networks. An actor in a given network experiences multiple cultures due to their affiliations with multiple communities. Due to the nature of the network, the information is oftentimes is oftentimes incomplete, because there is not a central authority to control or interpret the information.

Contrary to common belief, these features of networks may be useful for the users to help share their knowledge and opinions with each other. Moreover, the diversity and procedural 
uncertainty of an idea generation process such as high levels of ambiguity and frequent distractions typically hinder the process rather than promoting the problem-solving process.

The remainder of this paper is organized as follows. Issues from other studies and this study's theoretical model are discussed in the second section. Empirical results and their implications for system management are presented in the fifth section, followed by research conclusions. In the complex online environment, there are various problems with verifying the utility of content. In this study, it was hypothesized that, paradoxically, ambiguity, switching, multiplexity, and cohesiveness are the main drivers of social innovation online due to the diversity of interpretations and provocative features.

\section{Theoretical Background}

\subsection{Societal Innovativeness in OSIC}

This study investigates the online social innovation landscape. We observed the phenomenon of the users within the online community who were willing to contribute their local knowledge and resources to try and help solve social problems. OSIC is an platform in which this phenomenon occurs. The term "OSIC" has emerged to define the contributions being made by the deployment of users' excess resources in the forms of micro-volunteering and microactions. OSIC often facilitates the social media to provide a certain amount of fluidity and to help share the information. Additionally, OSIC have boundary-spanned interactions and goal ambiguity. In the past environments that helped promote innovation were considered to be static spaces. However, OSIC offers dynamic environments [11]. In spite of the lack of clarity the users may face in OSIC environments, if they are able to have a shared understanding about the social problem they are facing, they should be able to solve it in a collective behavior [4].

Social innovation is a citizen-centered collective behavior that solves societal challenges without government or companies support [5]. Unlike in the case of business innovation, government organizations may accumulate knowledge from the public to provide beneficial solutions [4][5]. To date, there has been a lack of research that deals directly with the elements of social innovativeness in OSIC platforms, which is defined as collective behavior that is voluntarily organized by citizens of urban societies [2][5]. Studies that are remotely related to social innovation can be classified into three categories. First, human computer interaction (HCI) and computer support cooperative work (CSCW) studies have focused on the technological features of online communities related to social innovation. Second, management and organization studies have focused on motivation or social-psychological factors of co-creation activities based on an open innovation model, in which an ordinary person can participate [3]. Numerous empirical and theoretical open innovation studies have been conducted that explored the possibilities of applying micro-organization theory to the context of online collaboration movement [12][13][14]. More specifically, open innovation studies have determined which environmental conditions encourage idea generation [15]. Third, micro-action and -volunteering studies have provided concrete examples of online social innovation communities [16]. For instance, Amichai-Hamburger and other researchers studied collective behaviors based on cooperative identities in geographic information-sharing communities [13][15]. Some scholars focused on re-evaluating the of effects generated in online pro-social communities [15][16]. These studies found that some of the factors that were significant in offline community idea generation were also significant to online community idea generation. 
As mentioned above, prior studies have focused on the complexity, fluidity, or boundaries of communities and examined which factors of social environments influence individuals. Many studies have observed social contagions and social learning in the online environment [16][17]. Moreover, some studies have observed the users who use online platforms, but they did not focus on the outcomes or creative solutions that were produced on the platforms or what factors motivated these results. The technological advancements in the mobile technology and networks have helped propelled the users to access the online communities via the mobile devices. Studies have found that users are likely to show interest in more than one topic, so a more theoretical and practical framework is necessart to observe this phenomenon.

\subsection{Network and Communication Framework}

In this study, we focus on the online curation platform for social innovation. Since the late 2000s, crowdsourcing, a process through which governments and companies collaborate with larger social groups, has gain a momentum within the ideation platforms for developing various types of infrastructure, services, and social products. IDEO, a global consulting firm, and many other companies have provided customizable design kits to help entrepreneurs, communities, and innovators reach and share with one another to help poor and vulnerable communities. Using social networks theory instead of organizational innovation theory to define the platform would be more appropriate to describe the platform because the former can explain some social trends such as free dicussion, participation, and convergence [7][8].

The main framework of this study is the network and communication framework, which originated from the social network theory. It focuses on the relationships between the actors, while the network and communication framework focuses on the social value, language, and culture. According to White et al. [10], past studies have focused on the sociolinguistic aspects of online interactions amongst community participants and found certain particular attributes and norms of the language used by digital nomads. The network and communication framework considers all of the social phenomenon as a constructed reality through discussion between and consensus among participants [18][19]. Another feature of the network and communication framework is complexity. People who have overlapping relationships communicate with each other [19][20]. Sociologists have argued that the existence of interpersonal and inter-domain networks that exist through the concept of netdom make up the reality of the world today and that the discussions and communications in this space are often intersubjective [21]. Since the inception of an idea, it is difficult to produce a solution where all of the participants concur with the solution [22]. OSIC platforms are not developed by government or companies to generate profit, so they may not have clear objectives in their initial stages, but instead they focus on allowing participants to create and share ideas and stories through the interwoven relationships that they have to establish [10][11].

\subsubsection{Switching and Ambiguity}

White [17] states that netdoms, a networked domain composed of interwoven relationships between the networks of various social structures and their domains, have their own stories, themes, agendas, group identities, and problem-solving methods [23]. He pointed out how users switch between netdoms [8]. In order to understand how users switch within networks, the story and culture of each social structure must be understood [18][19]. Each netdom has its own communication style to maintain consistent relationships between participants and opinion leaders or other key members [20].

Similarly, sociologists have stressed the need to clarify ambiguous points so that users can better understand the thought processes behind different netdoms [23]. Other economic 
sociologists did not concentrate on the findings about information asymmetry and opportunistic behavior [19][20]. Ambiguity can cause individuals to interpret the same phenomenon in different ways. However, those who are capable of clarifying ambiguities are good at forming relationships within organizations. As a result, social network theory states that ambiguity is not a source of concern or uncertainty and that it should be embraced as a way to solve problems [18][24].

\subsubsection{Multiplexity and Cohesiveness}

An important dimension of the network and communication framework is the overlapping relationships between the individuals. Multiplexity exists when ties between individuals share mutual interests or relationships [18]. Given their nature, online curation platforms require participants to constantly question and validate the plausibility of ideas. As such, forming trust between participants and behaving transparency are vital parts of the process. Studies have found that individuals who encompasses multiplexity are less likely to share fraudulent or harmful information [20] and these people will refrain from acting in ways that may hinder their reputations within the network [10][12].

Conversely, the network and communication framework emphasizes the cohesiveness and mutiple networks that exist within organizations. Cohesiveness is a vital factor in unifying communities [10][13]. Social psychologists have found that cohesiveness is closely related to social norms and community culture and it also helps online communiteis to share and solve problems. In the context of OSIC, cohesiveness can help small groups share their themes and ideas in certain ways within the social space. For instance, policy rules within groups are an example of how groups can maintain their cohesiveness.

Switching and ambiguity are attiributes of the contents, which is shared by participants in complex communities and networks and are particularly related to the uncertainty of content that exists within borderless internet communities, not traditional organizations such as OSIC platforms [19][23]. On the other hand, multiplexity and cohesiveness focus on participants' relationships within networks [13]. The aforementioned areas are focused on content and relationships.

\subsubsection{Experimentation, Interaction with Environments, and Societal Innovativeness}

Experimentation is an open platform that allows participants to help solve various problems [25][26]. The network theory argues that decisions made by individuals within hubs will influence innovation [27]. To illustrate this point, individuals within hubs have access to a wide spectrum of resources, which can give them a better perspectives and understanding of the information [20]. Thus, network environments are a vital part of the innovative capability that helps determine whether creative and experimental processes will be successful [26][27].

It is imperative to understand the value of prosocial behaviors within communities because this value depicts how participants interact with environments in innovative ways. Moreover, experimentation is important to network participants' cohesiveness and the process of developing and executing ideas.

Innovativeness within OSICs not only produces solutions to social problems, but it can also demonstrate how ideas can help to improve society. Innovativeness has been studied from both social network theory and behavioral science perspectives [28][29] and various studies have utilized different approaches. On the one hand, micro-organization theory states that organizational encouragement, openness, and challenges improve research creativity [26]. But on the other hand, Cyert and March [28] found that macro-organization theory treats innovativeness as a risk. Based on the discussions above, this study found that innovativeness 
was a source for ideas about how to help others and solutions to to problems [18][20].

\section{Research Model and Hypotheses}

Based on the discussions above, this study aimed to understand the communication elements found in social network communication theory (Fig. 1). As such, we attempt to define how the attributes of both content and participants affected community experiences and social relationships. In the research model, switching and ambiguity referred to content that is shared among participants, while they communicate within the network. Contrary to the organizational hierarchy, the OSIC platforms allow the community to collaborate and communicate without any boundaries or segmentations. Switching and ambiguity referred to users' uncertainty about shared knowledge or content [19][23]. Multiplexity and cohesiveness focus on the complex relationships that exist between participants [13]. The four dependent variables will affect the content attributes and social relationships within the community, and the communication entails the openness, quality, and tone of the activity in the OSIC platform.

In the context of OSIC platforms, experimentation and environmental interaction refer to how OSIC participants are willing to solve social problems and how socially innovative actions can help solve problems. The collective experience and continuous effect of communication create new ideas and help to maintain connections between network communication and innovativeness. Societal innovativeness is the outcome of ideas created to solve social problems.

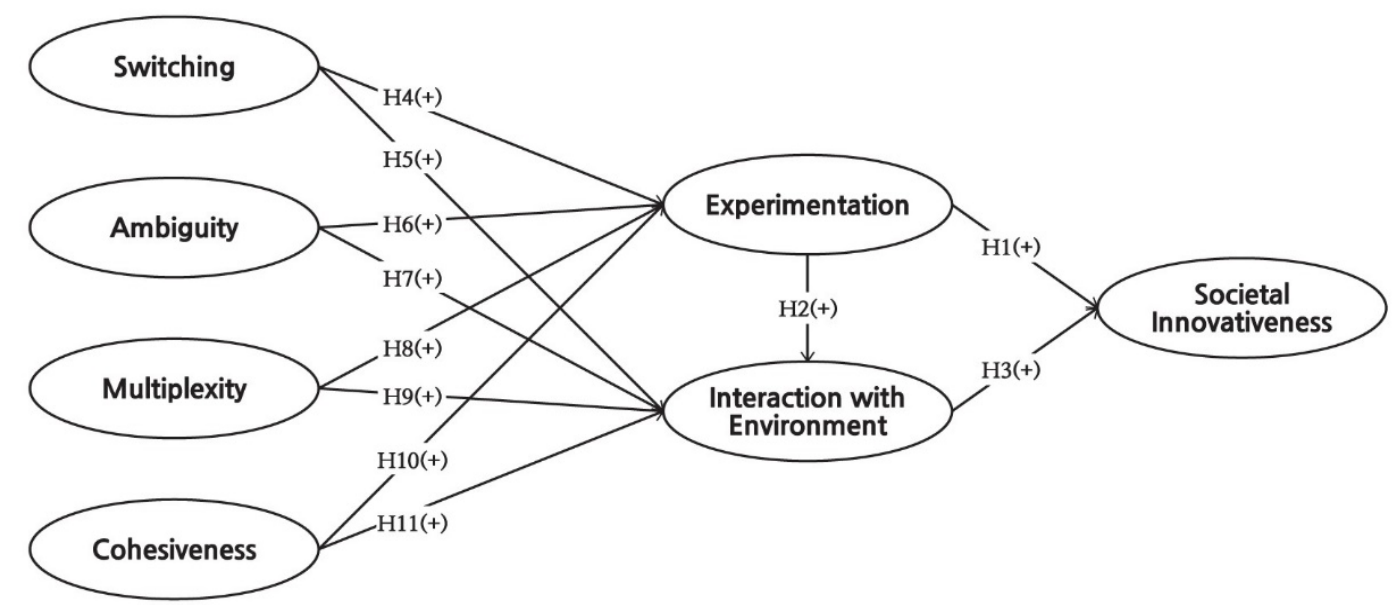

Fig. 1. Research Model

\subsection{Innovative Outcomes of Experimentation}

Experimentation refers to the discovery of new sets of experiences or patterns that have not been defined in the past [28][29]. Often users are not bound by rules or responsibilities in the online environment and instead form new social structures there. As such, efficiency emerges from the combination of structured workflows and task assignments [27]. Many studies have pointed out how a lack of comprehension of problems can help enhance the individual's creativity [25]. Finding endogenous factors by accident or serendipity is a necessary part of the innovative process [28].

Societal innovativeness is similar to perceived creativity, as it entails how valuable users 
think their contributions are [16]. Online environments that rely on users to voluntarily make social contributions should focus on how those contributions are meaningful [12][13], which shows how significant users are to subjective satisfaction [14]. Based on the previous studies, systematic structures that include an open atmosphere and open interpersonal communication between the online community members can help generate creativity. Both the empowerment and managerial discretion can be found in official and unofficial online communities [15].

Hypothesis 1. Experimentation will have a positive effect on the societal innovativeness.

The mediation between different actors is vital for shaping environments and interactivity that can generate innovative ideas [13]. Some scholars stressed the significance of internal and external stakeholders of OSIC platform [12][15]. Moreover, it is vital that users be able to freely communicate and share information between themselves to solve problems [11]. The unexpected solutions discovered during these communications are an imperative part of encouraging users to share their experiences [10].

Hypothesis 2. Experimentation will have a positive effect on the interaction with the environment.

\subsection{Environmental Interaction in the Context of Social Innovativeness}

User environmental interaction can generate solutions to social problems [11]. Constant discussions and collaborative activities help to generate progress. Projects such as human-centered design (HCD) and crowdsourcing require users to provide quality assurance for each of their projects [13][14]. Retaining an open line of communication is key to improving the quality of the final results. The interactions within environments improves ideation process by providing control and commitment, which helps social innovations to be more flexible and legitimate [20]. Concrete community designs encourages everyone to participate in developing creative designs [11].

Hypothesis 3. Increasing the interactions with the environment will have a positive relationship to the Societal Innovativeness.

\subsection{Switching, Experimentation, and Environmental Interaction}

White [17] stated that the majority of actors associate themselves with more than one group and that individuals can have diverse sets of personal traits. As such, switching occurs when users initiate new relationships given their complex nature. In online community environment, complex relationships and information exchanges determine which decisions are made [14][15]. Social network studies argued how important identifying and improving the blind are. Such improvement permits users to search across a broader range of topics more efficiently, which allows for more systematic observation.

Hypothesis 4. Improving the switching will have a positive relationship with the experimintation.

Switching constitutes both changes in theme and story switching. Actors use structured signaling methods to communicate with each other [10]. Networks will progressively connect new nodes to groups, so new themes will emerge and change the styles of discussions. Online community design requires that actors have complex interactions [15][16]. Switching crosses 
boundaries while encouraging environmental interaction.

Hypothesis 5. Switching will increase the number of environmental interactions.

\subsection{Ambiguity, Experimentation, and Environmental Interaction}

Ambiguity occurs when there is insufficient amount of information to make a decision [18][19]. As mentioned above, there are many domains in which ambiguity arises between actors. However, it is impossible to attempt to resolve ambiguity, so it should be utilized instead [8]. Users communicate with each other on online social platforms across a wide array of topics and discussions [30]. These interactions have the potential to create ambiguity regarding ideas and collaborative activties, but this ambiguity can actually help motivate users and make them more creative [15][16].

Hypothesis 6. An ambiguity that has been fully aware will help improve the experimentation.

Ambiguity allows members of social innovation communities to freely converse with their peers without being bound by particular ways of communication. Incorporating lessons from evolutionary psychology into information system environments will enhance the richness of media while expanding the channels and tasks of specific fields [14][17]. For these reasons, we believe that having numerous paths for a project or different sources of connectivity will have a positive relationship with the innovative capability.

Hypothesis 7. An ambiguity that has been fully aware will help improve the interactions with the environment.

\subsection{Multiplexity and Cohesiveness during the Innovative Process}

Multiplexity refers to two or more sets of relationship that individuals share, so multiplexity is a product of the number of interactions and relationships between individuals [20][22]. Researchers have examined network properties to reduce these engagements to increase the quality of the discussions [19]. Sociologists stated that reciprocity will increase when actors are in close proximity to each other. White [17] stated that actors communicating their overlapping thoughts may improve the creative process. Thus, this study presumed that multiplexity strongly influenced experimentations.

\section{Hypothesis 8. The rise in Multiplexity may help improve the experimentations.}

When the actors that are densely connected within the social network, the actors' mutual sense of solidarity and community spirit increases. Forming a common ground is essential for forming an in-group identity within the OSIC environment [20]. OSICs provide tools that help participants to exchange ideas [15]. In order to solve societal problems, OSIC should enable the users to reconstruct the spaces. This study presumed that overlapping participant relationships helped to improve communication and information flow.

Hypothesis 9. The increase in multiplexity will have a positive relationship to the interactions with the environment.

The difference in both the network position and strength determines structural inequality 
[18], which can influence cohesiveness. Cohesiveness can reduce innovativeness, but it can also increase it as a tertius iungens [19]. Interdependent participants can jointly solve problems to reach shared goals [18][19]. In this study, we posit that cohesiveness is not necessarily a hindrance and could be strategically implemented.

Hypothesis 10. Cohesiveness has a positive relationship to the experimentation.

As mentioned above, we suggest that structural inequality cannot be viewed simply as a problem [19]. According to White [17], asymmetric and imbalanced information flow can encourage searching behaviors. Sociologists stated that the complex nature of networks and structural inequality can provoke the participants to interct with their environments [22][23] [30]. Within the OSIC, the environments, discussions, and information are provided through them may tend to be of a quality promoted by lead users and offline leaders. We posit our next hypothesis based on these types of inequalities.

Hypothesis 11. Cohesiveness has a positive relationship with the interactions with the environment.

\section{Method and Results}

\subsection{Data Collection}

In this study, we focused on 'Design Kit' for the OSIC platform in the online community. Since IDEO introduced the HCD Connects, around 50,000 users have participated on the platform. The Design Kit allows anyone to easily participate in the communities to help solve problems. For instance, the HCD Toolkit helped improve the water distribution system management system in Rwanda and ideas for saving the trees in Siberia have been effective and constructive.

We conducted an online survey on the Design Kit users. We measured the 21 major items and demographic items using the 7-point Likert scales. prior to conducting the survey, a cognitive review was performed by a linguistic expert who is fluent in Korean and English. A pilot test was conducted on 20 Design Kit users to correct errors in the survey. The survey was conducted from March 1-14, 2016. We selected a random sampling method on the individuals and groups who participated in projects that utilized the Design Kit. We performed random sampling by sending an email to the participant's email address, which were provided with the help of service administrators. A total of 471 survey responses came from users in a many different countries. After removing the 73 surveys that were identified as outliers, we had a total of 398 responses. The measurement items for the table are depicted in Table $\mathbf{1}$.

Table 1. Measurement items

\begin{tabular}{|c|}
\hline Constructs / Sources / Measurement Items \\
\hline \hline Switching / self-developed Items, referred definitions of White et al. [10]; Mische \& White [22] \\
\hline (sw1) Experience switching of theme when platform owner transit main agenda \\
(sw2) Communication styles are changed when thematic changes occur \\
(sw3) Role and task are changes when thematic changes occur \\
\hline Ambiguity / self-developed items, referred definitions of White et al. [10]; White [17] \\
\hline (amb1) The themes are difficult to define characteristics in Design Kit \\
(amb2) The information is not enough to decide whether it is true or not \\
(amb3) Ideas could be interpreted in various ways \\
\hline
\end{tabular}




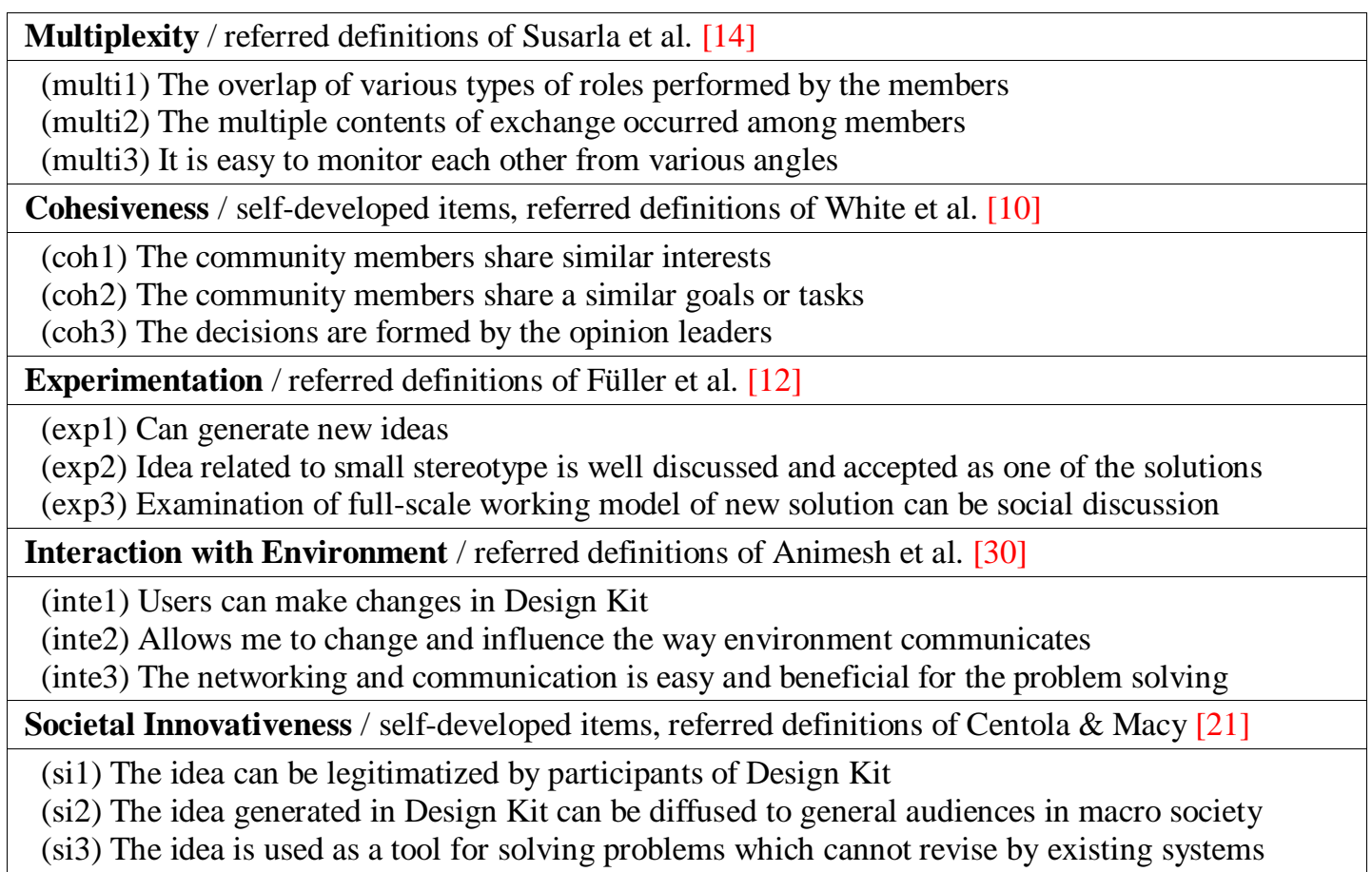

According to Podsakoff et al. [31], experimental studies that measure both the independent and dependent variables on the same subject at the same time are subject to a common method bias. In order to minimize the common method bias from occuring, procedural and statistical control methods were implemented in the experimental model. Survey measures and variables were provided from the early stages of creating the survey.

The Harman's Single Factor test was conducted to check the common method variance indicated that the limit of 0.25 was not exceeded [32][33]. The sample population comprised of 240 males and 158 females. And 280 members of the sample population (70\%) were between the ages of 20 and 30 . Approximately $83 \%$ of the respondents used OSIC platforms less than three hours per week on average as depicted in Table 2.

Table 2. Demographic data of participants ( $\mathrm{N}=398$ )

\begin{tabular}{|c|c|c|}
\hline \multicolumn{2}{|c|}{ Demographics } & Frequency (\%) \\
\hline \hline \multirow{2}{*}{ Gender } & Female & $158(39.7)$ \\
\cline { 2 - 3 } Age & Male & $240(60.3)$ \\
\hline \multirow{3}{*}{ Occupation } & $20-30$ & $280(70.4)$ \\
\cline { 2 - 3 } & $30-40$ & $69(17.3)$ \\
\cline { 2 - 3 } & 40 -order & $49(12.3)$ \\
\cline { 2 - 3 } & Student & $232(58.3)$ \\
\cline { 2 - 3 } & Working & $148(37.2)$ \\
\hline \multirow{3}{*}{ Education } & Unemployed & $18(4.5)$ \\
\cline { 2 - 3 } & High School & $25(6.3)$ \\
\cline { 2 - 3 } Time spent in OSIC & Undergraduate & $263(66.1)$ \\
\cline { 2 - 3 } (Hours per week) & Graduate & $110(27.6)$ \\
\cline { 2 - 3 } & Less than 1 & $123(30.9)$ \\
\cline { 2 - 3 } & $1-3$ & $210(52.8)$ \\
\hline
\end{tabular}




\subsection{Data Analysis and Results}

We applied the structural equation modeling (SEM) to examine the empirical analysis. LISREL 8.80 software that was capable of conducting covariance-based SEM was used to conduct a maximum-likelihood analysis. The structural SEM model used in this study is based on equation (1). In equation (1), The $\eta$ variables are the endogenous latent variables: $\eta_{1}$ is social innovativeness, $\eta_{2}$ is experimentation, and $\eta_{3}$ is environmental interaction. The $\xi$ variables are the four exogenous latent variables and $\beta$ is the endogenous latent variable that links the matrix coefficient $\gamma$, and the exogenous and endogenous latent variables and errors, $\zeta$.

$$
\begin{gathered}
\eta_{1}=\beta_{12} \eta_{2}+\beta_{13} \eta_{3}+\zeta_{1} \\
\eta_{2}=\gamma_{21} \xi_{1}+\gamma_{22} \xi_{2}+\gamma_{23} \xi_{3}+\gamma_{24} \xi_{4}+\zeta_{2} \\
\eta_{3}=\beta_{32} \eta_{2}+\gamma_{31} \xi_{1}+\gamma_{32} \xi_{2}+\gamma_{33} \xi_{3}+\gamma_{34} \xi_{4}+\zeta_{3}
\end{gathered}
$$

The relationship between the experimental model's exogenous and endogenous variables was established by the observed and latent variables, as shown in equation (2) and (3).

$$
\begin{gathered}
X_{(q \times 1)}=\Lambda_{\chi(q \times n)} \times \xi_{(n \times 1)}+\delta_{(q \times 1)} \\
Y_{(p \times 1)}=\Lambda_{y(p \times n)} \times \eta_{(m \times 1)}+\varepsilon_{(p \times 1)}
\end{gathered}
$$

The exogenous latent variable, $\Lambda x$, and endogenous latent variable, $\Lambda y$, were connected to the endogenous latent variable's vector $\xi$, and the exogenous latent variable's vector $\eta$. $\delta$ and $\varepsilon$ are the error vector magnitudes.

\subsubsection{Reliability and Validity of the Measures}

Prior to evaluating the measurement model, we conducted an exploratory factor analysis (EFA) with principal component analysis and varimax rotation using SPSS 18.0 to extract latent (independent) variables, as shown in Table 3. Seven factors were extracted and their variances, factor loadings, and eigenvalues were calculated. The Each factor's eigenvalue was above 1.0.

Table 3. Results of exploratory factor analysis (factor loadings and cross factor loadings)

\begin{tabular}{|c|c|c|c|c|c|c|c|}
\multirow{2}{*}{ Item } & \multicolumn{7}{|c|}{ Component } \\
\cline { 2 - 8 } & $\mathbf{1}$ & $\mathbf{2}$ & $\mathbf{3}$ & $\mathbf{4}$ & $\mathbf{5}$ & $\mathbf{6}$ & $\mathbf{7}$ \\
\hline \hline Perf2 & .837 & .118 & .063 & .280 & .211 & -.010 & .151 \\
\hline Perf1 & .828 & .143 & .094 & .264 & .184 & .027 & .140 \\
\hline Perf3 & .810 & .149 & .055 & .194 & .275 & -.054 & .115 \\
\hline Swit2 & .099 & .899 & .054 & .023 & .127 & .032 & .126 \\
\hline Swit3 & .159 & .849 & .191 & .037 & -.011 & .019 & .139 \\
\hline Swit1 & .080 & .842 & -.003 & .082 & .128 & .108 & -.007 \\
\hline Multi2 & .056 & .150 & .891 & .124 & .097 & .122 & .068 \\
\hline Multi3 & .088 & .113 & .882 & .145 & .079 & .170 & .127 \\
\hline Multi1 & .051 & -.030 & .710 & -.037 & .233 & .246 & .230 \\
\cline { 2 - 8 } Inter1 & .212 & -.028 & .013 & .845 & .062 & .007 & .107 \\
\hline
\end{tabular}




\begin{tabular}{|c|c|c|c|c|c|c|c|}
\cline { 2 - 7 } Inter2 & .204 & .212 & .215 & .778 & .153 & -.050 & .135 \\
\hline Inter3 & .265 & .014 & .057 & .772 & .189 & -.024 & .233 \\
\hline Exp1 & .165 & .079 & .065 & .164 & .832 & .076 & .212 \\
\hline Exp2 & .278 & .150 & .115 & .118 & .767 & .127 & .278 \\
\hline Exp3 & .250 & .081 & .257 & .124 & .753 & -.099 & .033 \\
\hline Coh2 & .091 & .001 & .116 & .017 & .022 & .869 & .049 \\
\hline Coh1 & -.016 & .022 & .162 & -.050 & .047 & .804 & .084 \\
\hline Coh3 & -.102 & .128 & .130 & -.011 & -.003 & .802 & .028 \\
\hline Ambi3 & .144 & .076 & .072 & .156 & .103 & .141 & .809 \\
\hline Ambi1 & .220 & -.001 & .150 & .157 & .123 & .079 & .785 \\
\cline { 2 - 8 } Ambi2 & .000 & .202 & .165 & .118 & .213 & -.035 & .701 \\
\hline Eigenvalue & 2.508 & 2.477 & 2.377 & 2.270 & 2.242 & 2.226 & 2.144 \\
\hline Variance (\%) & 11.943 & 11.795 & 11.319 & 10.811 & 10.674 & 10.601 & 10.211 \\
\hline Cum. Variance (\%) & 11.943 & 23.738 & 35.057 & 45.868 & 56.542 & 67.143 & 77.355 \\
\hline
\end{tabular}

In the next phase, confirmatory factor analysis (CFA) was employed to calculate the standardized factor loadings. Then, we tested the reliability and validity of the measures shown in Table 4.

Table 4. Results of convergent validity and reliability (measurement model statistics)

\begin{tabular}{|c|c|c|c|c|c|c|}
\hline Construct & Item & Std. loading & $t$-value & AVE & CR & $\alpha$ \\
\hline \multirow{3}{*}{1} & Perf1 & 0.89 & - & \multirow{3}{*}{0.770} & \multirow{3}{*}{0.907} & \multirow{3}{*}{0.905} \\
\hline & Perf2 & $0.92 * *$ & 26.16 & & & \\
\hline & Perf3 & $0.82 * *$ & 21.31 & & & \\
\hline \multirow{3}{*}{2} & Swit1 & 0.74 & - & \multirow{3}{*}{0.708} & \multirow{3}{*}{0.878} & \multirow{3}{*}{0.868} \\
\hline & Swit2 & $0.95 * *$ & 23.21 & & & \\
\hline & Swit3 & $0.82 * *$ & 18.88 & & & \\
\hline \multirow{3}{*}{3} & Multi1 & 0.66 & - & \multirow{3}{*}{0.548} & \multirow{3}{*}{0.783} & \multirow{3}{*}{0.852} \\
\hline & Multi2 & $0.83^{* *}$ & 22.03 & & & \\
\hline & Multi3 & $0.72 * *$ & 23.93 & & & \\
\hline \multirow{3}{*}{4} & Inter1 & 0.74 & - & \multirow{3}{*}{0.636} & \multirow{3}{*}{0.840} & \multirow{3}{*}{0.833} \\
\hline & Inter2 & $0.82^{* *}$ & 15.09 & & & \\
\hline & Inter3 & $0.83^{* *}$ & 15.31 & & & \\
\hline \multirow{3}{*}{5} & Exp1 & 0.82 & - & \multirow{3}{*}{0.654} & \multirow{3}{*}{0.848} & \multirow{3}{*}{0.839} \\
\hline & Exp2 & $0.91^{* *}$ & 19.20 & & & \\
\hline & Exp3 & $0.68 * *$ & 14.29 & & & \\
\hline \multirow{3}{*}{6} & Coh1 & 0.76 & - & \multirow{3}{*}{0.595} & \multirow{3}{*}{0.815} & \multirow{3}{*}{0.791} \\
\hline & Coh2 & $0.83^{* *}$ & 17.89 & & & \\
\hline & Coh3 & $0.72 * *$ & 15.21 & & & \\
\hline \multirow{3}{*}{7} & Ambi1 & 0.78 & - & \multirow{3}{*}{0.537} & \multirow{3}{*}{0.775} & \multirow{3}{*}{0.761} \\
\hline & Ambi2 & $0.64^{* *}$ & 12.79 & & & \\
\hline & Ambi3 & $0.77^{* *}$ & 16.07 & & & \\
\hline
\end{tabular}


Cronbach's alpha and composite reliability (CR) score were above 0.6, and the average variance extracted (AVE) values were between 0.537 and 0.770 . Both the CR and AVE have a greater threshold value of 0.5 , and all of the standardized factor loadings were also acceptable and significant at the $\mathrm{p}=0.05$ level of convergent validity.

The discriminant validity was established by investigating the correlation values between the latent variables, which needed to be less than 0.6, and the square roots of AVE should have been above the correlation for a pair of constructs. The results shown in Table 5. represents that each indicator had a higher loading value than its construct [33].

Table 5. Correlations between latent variables and discriminant validity

\begin{tabular}{|c|c|c|c|c|c|c|c|c|}
\hline Variables & $\mathbf{M}$ (SD) & $\mathbf{1}$ & $\mathbf{2}$ & $\mathbf{3}$ & $\mathbf{4}$ & $\mathbf{5}$ & $\mathbf{6}$ & $\mathbf{7}$ \\
\hline \hline 1 & $4.66(1.40)$ & $\mathbf{0 . 8 0 9}$ & & & & & & \\
\hline 2 & $4.22(1.56)$ & $0.457^{* *}$ & $\mathbf{0 . 7 3 3}$ & & & & & \\
\hline 3 & $5.11(1.33)$ & $0.414^{* *}$ & $0.419^{* *}$ & $\mathbf{0 . 7 9 7}$ & & & & \\
\hline 4 & $4.74(1.44)$ & $0.557^{* *}$ & $0.395^{* *}$ & $0.566^{* *}$ & $\mathbf{0 . 8 7 7}$ & & & \\
\hline 5 & $3.95(1.46)$ & $0.280^{* *}$ & $0.251^{* *}$ & $0.199^{* *}$ & $0.310^{* *}$ & $\mathbf{0 . 8 4 1}$ & & \\
\hline 6 & $5.45(0.90)$ & $0.104^{*}$ & $0.172^{* *}$ & -0.008 & 0.008 & $0.134^{* *}$ & $\mathbf{0 . 7 7 1}$ & \\
\hline 7 & $5.29(1.11)$ & $0.380^{* *}$ & $0.370^{* *}$ & $0.254^{* *}$ & $0.232^{* *}$ & $0.228^{* *}$ & $0.360^{* *}$ & $\mathbf{0 . 7 4 0}$ \\
\hline
\end{tabular}

Note. 1 Experimentation, 2 Ambiguity, 3 Interaction with environment, 4 Societal Innovativeness, 5 Switching, 6 Cohesiveness, 7 Multiplexity $\left({ }^{*} \mathrm{p}<.05,{ }^{* *} \mathrm{p}<.01\right.$, Bold number shows the square roots of AVE)

Before performing structural analysis, we examined both the measurement and structural model to observe their fit indicies, as shown in Table 6. All fit index values complied with recommended criteria [33], which indicated that the experimental model was feasible.

Table 6. Results of model fit Indices

\begin{tabular}{|c|c|c|c|}
\hline \multirow{2}{*}{ Index } & \multicolumn{2}{|c|}{ Results of Fit Index } & Recommended Value \\
& Measurement Model & Structural Model & - \\
\hline chi-square & 436.82 & 440.78 & - \\
\hline DF & 168 & 172 & $<3.0$ \\
\hline Normed chi-square & 2.60 & 2.56 & $<0.08$ \\
\hline RMSEA & 0.063 & 0.063 & $<0.5$ \\
\hline RMR & 0.11 & 0.12 & $>0.90$ \\
\hline CFI & 0.97 & 0.97 & $>0.90$ \\
\hline GFI & 0.91 & 0.90 & $>0.80$ \\
\hline AGFI & 0.87 & 0.87 & $>0.90$ \\
\hline NFI & 0.95 & 0.95 & $>0.90$ \\
\hline NNFI & 0.96 & 0.96 & \\
\hline
\end{tabular}

\subsubsection{Result of Structural Model Analysis}

The purpose of this study was to determine how network-communication characteristics affected the innovativeness of OSIC. The effect of the variables was tested by conducting structural analysis to determine whether the data would require the hypotheses to be rejected. The findings represented all hypotheses were retained, except for H5, H10 (See Table 7 and Fig. 2). The models' R-squared values, representing their explanatory power, were $0.40,0.63$, and 0.55 , respectively. 
Table 7. Results of hypothesis test

\begin{tabular}{|c|c|c|c|c|c|}
\hline Hypothesis & Path & Path coefficient & $\boldsymbol{t}$-value & S.E. & Result \\
\hline \hline H1 & EXP $\rightarrow$ PER & $0.38^{* * *}$ & 7.21 & 0.052 & retain \\
\hline H2 & EXP $\rightarrow$ INT & $0.24^{* * *}$ & 3.47 & 0.070 & retain \\
\hline H3 & INT $\rightarrow$ PER & $0.48^{* * *}$ & 8.45 & 0.057 & retain \\
\hline H4 & SWI $\rightarrow$ EXP & $0.17^{* * *}$ & 3.27 & 0.051 & retain \\
\hline H5 & SWI $\rightarrow$ INT & 0.041 & 0.76 & 0.053 & reject \\
\hline H6 & $\mathrm{AMB} \rightarrow$ EXP & $0.48^{* * *}$ & 7.55 & 0.063 & retain \\
\hline H7 & $\mathrm{AMB} \rightarrow \mathrm{INT}$ & $0.36^{* * *}$ & 4.75 & 0.076 & retain \\
\hline H8 & MUL $\rightarrow$ EXP & $0.14^{*}$ & 2.46 & 0.058 & retain \\
\hline H9 & MUL $\rightarrow$ INT & $0.16^{* *}$ & 2.58 & 0.061 & retain \\
\hline H10 & $\mathrm{COH} \rightarrow$ EXP & 0.00034 & 0.0061 & 0.056 & reject \\
\hline H11 & $\mathrm{COH} \rightarrow \mathrm{INT}$ & $-0.15^{*}$ & -2.54 & 0.059 & reject \\
\hline
\end{tabular}

Note. INT(Interaction with environment), EXP(Experimentation), AMB(Ambiguity), SWI(Switching), PER(Societal Innovativeness), $\mathrm{COH}\left(\right.$ Cohesiveness), MUL(Multiplexity) $\left({ }^{*} \mathrm{p}<.05,{ }^{* *} \mathrm{p}<.01,{ }^{* * *} \mathrm{p}<.001\right)$

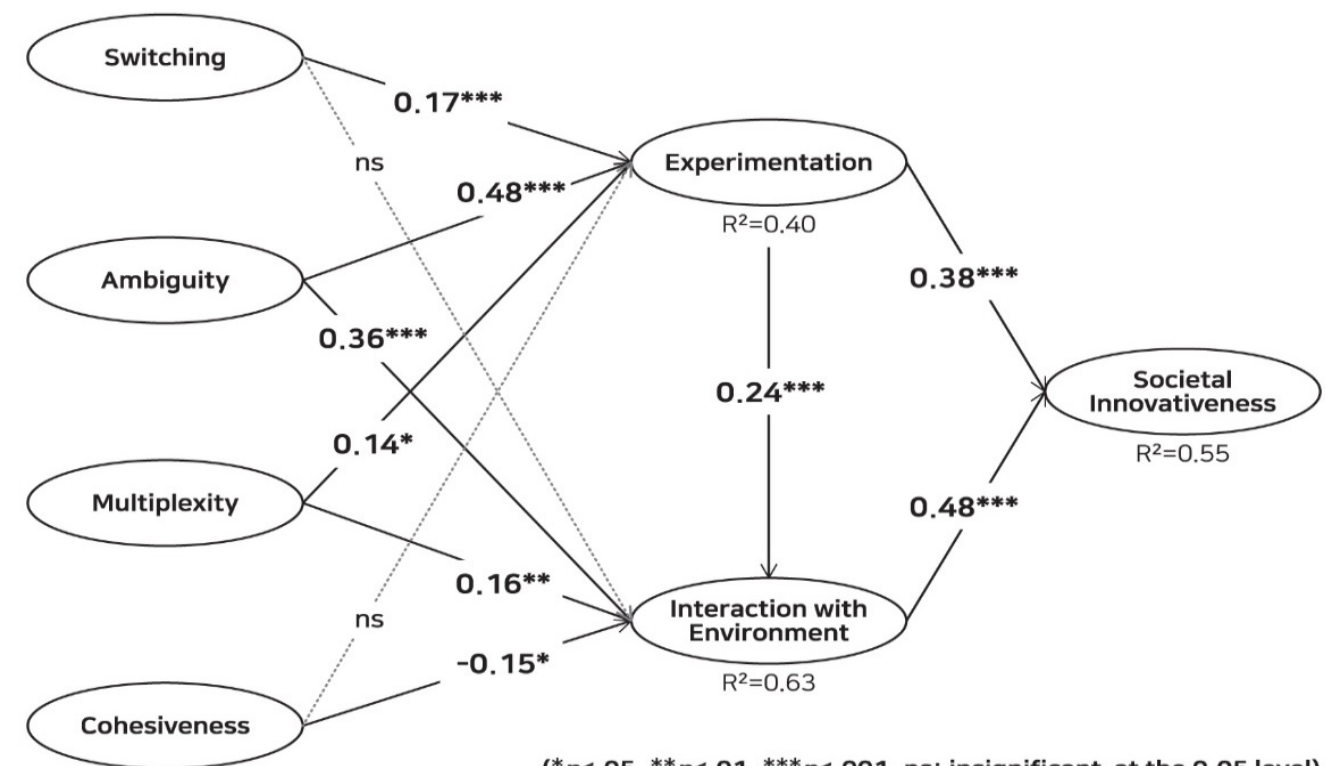

$\left({ }^{*} \boldsymbol{p}<.05,{ }^{* *} \boldsymbol{p}<.01,{ }^{* * *} \boldsymbol{p}<.001, \mathrm{~ns}:\right.$ insignificant at the 0.05 level)

Fig. 2. Structural model for hypotheses

Of the four constructs related to network and communication factors, ambiguity, switching, and multiplexity were found to improve experimentation and interactivity. The results show network and communication features improve OSIC innovativeness. However, we were unable to find any correlations between the switching and interaction with the environment. There was no significant relationship between the cohesiveness and experimentations. Also, cohesiveness had a negative relationship to the interaction with the environment. This can be interpreted as the construct duality of the cohesiveness. As mentioned above, it was presumed that cohesiveness could help users overcome structural inequalities, but the results indicated that structural inequality reduced the number of environmental interactions. 
Table 8. Results of Mediating Effect Test

\begin{tabular}{|c|c|c|c|c|c|c|c|c|c|}
\hline & M1 & M2 & M3 & M4 & M5 & M6 & M7 & M8 & M9 \\
\hline Criterion & EXP & INT & PER & INT & PER & PER & PER & PER & PER \\
\hline \multicolumn{10}{|l|}{ IV } \\
\hline SWI & Ns & 0.16** & $0.19 * * *$ & & & & $0.12 *$ & $0.15 * * *$ & 0.10* \\
\hline AMB & $0.47 * * *$ & $0.48 * * *$ & $0.40 * * *$ & & & & $0.18 * *$ & $0.14 *$ & ns \\
\hline $\mathrm{COH}$ & $-0.15 *$ & ns & ns & & & & ns & $\mathrm{ns}$ & ns \\
\hline MUL & $0.20 * * *$ & $0.15^{*}$ & ns & & & & ns & ns & ns \\
\hline \multicolumn{10}{|l|}{ MV } \\
\hline $\operatorname{EXP}(1)$ & & & & $0.51 * * *$ & $0.62 * * *$ & & $0.48 * * *$ & & $0.36 * * *$ \\
\hline INT(2) & & & & & & $0.67 * * *$ & & $0.56 * * *$ & $0.48 * * *$ \\
\hline $\mathrm{R}^{2}$ & 0.33 & 0.39 & 0.28 & 0.26 & 0.38 & 0.44 & 0.42 & 0.48 & 0.56 \\
\hline $\begin{array}{l}\text { Effect size } \\
\left(f^{2}\right) \\
\text { Pseudo } \mathrm{F}\end{array}$ & & & & & & & $\begin{array}{c}\text { Medium } \\
0.24 * * * \\
94.08\end{array}$ & $\begin{array}{c}\text { Large } \\
0.38 * * * \\
148.96\end{array}$ & $\begin{array}{c}\text { Large } \\
0.64 * * * \\
250.24\end{array}$ \\
\hline
\end{tabular}

Note. INT(Interaction with environment), EXP(Experimentation), AMB(Ambiguity), $\mathrm{COH}(\mathrm{Cohesiveness),}$ SWI(Switching), PER(Societal Innovativeness), MUL(Multiplexity) $\left({ }^{*} \mathrm{p}<.05,{ }^{* *} \mathrm{p}<.01,{ }^{* * *} \mathrm{p}<.001\right.$, ns: insignificant at the 0.05 level). M1(IV $\rightarrow$ MV1), M2(IV $\rightarrow$ MV2), M3(IV $\rightarrow$ DV), M4(MV1 $\rightarrow$ MV2), M5(MV1 $\rightarrow$ DV), M6(MV2 $\rightarrow$ DV), M7 (IV,MV1 $\rightarrow$ DV), M8(IV,MV2 $\rightarrow$ DV), M9(IV,MV1,MV2 $\rightarrow$ DV)

This study used mediating variables to extend the theoretical framework, as shown in Table 8. The results indicated that the mediating variables, experimentations, and environmental interactions had considerable mediating effects on network-communication and OSIC platform innovativeness. Notably, the size of the mediating effect was large $\left[f^{2}=\right.$ (full model $R^{2}$ - reduced model $\left.R^{2}\right) /\left(1\right.$ - full model $\left.\mathrm{R}^{2}\right)$, pseudo $F=\left(f^{2} \times\right.$ sample size $)$ - (number of full model independent variables -1$\left.), \mathrm{F}_{\mathrm{CV}}=\mathrm{F}_{(1, \mathrm{~N}-\mathrm{K})}\right]$ and statistically significant.

\section{Conclusion}

Based on the theoretical framework of the network and communication theory, we explored the environmental characteristics of the OSIC platforms. we conceptualized the notion of societal innovativeness as the outcome of socially meaningful and innovative ideation. As a Schumpeterian perspective suggest, innovation is not limited to managerial objectives and can be extended to social ends to meet human needs [21]. We set ambiguity, switching, multiplexity, cohesiveness as the four main variables of this study that can be inferred to facilitator of innovative capabilities and societal innovativeness. Although these factors have been regarded as the sources of uncertainty, in this study they were treated as an environmental stimuli to which the users had to adapt [18]. Unlike offline organizations, the governance of online organizations' social systems is not centralized. Therefore, most of what takes place in online organizations arises from participants' shared understanding and not by a central authority which defines cultural and communication norms [20][34]. We confirmed the findings from the prior studies on switching, as the nature of OSIC and can be a motivator for users to participate in the collaborative communication. Switching, which is the change of social themes and roles, was also investigated as a facilitator of creativeness in OSICs. It was hypothesized that the dispersed cognition of participants was another benefit of OSICs' switching mechanism. Thematic change and diversity are expected to lead users to engage in generating diverse types of knowledge. 
However, this study contained a number of limitations. First, the real network structure among community participants was not considered due to the limitation of the survey design. With regard to survey analysis, private user information could not be acquired, including who each user was acquainted with on the platform, due to privacy issues and public sector controls [20][35]. In addition, the target population for the survey was relatively homogeneous. Second, measurement items were blended with existing theory and the context of social innovation to focus on constructing variables related to network theory over influencing variables. Nevertheless, in order to learn about the real impact of the online social innovation process, a real-world experiment is required to observe if group support has a mediating effect. Third, individual innovativeness should have been considered as a control variable. Although the survey was conducted among a limited population, the low and high internal innovativeness groups might have exhibited different behavioral patterns. A multi-group analysis may be required to develop more fruitful explanations about the social innovation process.

\subsection{Theoretical Implications}

Transparent information sharing is an important factor in the online innovation studies. Goal setting by means of sharing the same philosophies and objectives is also critical to effective communication among community members. However, the dark side of online networks arises due to group friction and conflict generated by frequent social interactions [10][20].

We inferred that ambiguity, switching, and multiplexity could be components of environmental dynamism would enact users to learn and adapt to innovative experience. When existing norms do not make corrections, users can enjoy ideation and opinion-sharing in ambiguous and switched communication structures. In addition, this study further explained social innovativeness by applying network and innovation constructs and measures. The notion of innovativeness has been applied theoretically and practically in many different contexts [29]. However, little research has been done on its effects from a social perspective. Furthermore, we attempted to explore the nature of the societal innovativeness and developing measurement, and theoretically expand it.

\subsection{Practical Implications}

The results from this study have practical implications for most businesses that utilize the online platform with the intention of involving users in a social innovation process. Practitioners must take into account the characteristics of the different types of ambiguities and switching dynamics when designing social innovation platforms. First, interaction among participants using incomplete information during idea generation is an important contributing factor to the creation of socially creative knowledge and systems. For instance, an idea that is proposed by a user may be ambiguous, but it can be expanded upon by the other users. These ambiguous ideas can be highlighted by formatting and by drawing attention to them.

In order to create a socially creative end product or service, allocating the participant's attention to a diverse set of information or selected information is important. The users' role in platforms primarily affects the social innovation process and the direction of the idea generation should be clearly navigated. Platform owners should highlight certain ideas or a thread of ideas to expand and focus on particular elements. The platform owner can also limit the number of incomplete ideas and prevent ideas from diverging from the topic at hand.

Additionally, it is possible to make clear design guides from the beginning of the discussion process for idea generation. Platforms are not directly influenced by their owners because of the number of participants and so should direct users' attention to a variety of stories to let users scan as many themes as they can. For example, system owners can increase the amount 
of information and incomplete ideas to increase the chances that social innovation community members will generate creative ideas.

This study sheds light on the environmental factor in the social innovation process and the relationship between elements of communication and societal innovativeness. The importance of social problem solving has been increasingly emphasized in the online media, such as social media, crowdsourcing platforms, and online-games. This study provides a small, but significant step towards developing innovative platforms. By studying online social innovation, ways to utilize telecommunication infrastructure to gather social funds and resources were discovered.

\section{References}

[1] S. Vujovic and J. Ulhøi, "Online innovation: the case of open source software development," European Journal of Innovation Management, vol.11, no.1, pp.142-156, 2008.

Article (CrossRef Link).

[2] S. Greengard, Samuel. "Following the crowd," Communications of the ACM, vol.54, no.2, pp.20-22, February, 2011. Article (CrossRef Link).

[3] G. Von Krogh and E. Von Hippel, "The promise of Research on Open Source Software," Management Science, vol.52, no.7, pp 975-983, July, 2006. Article (CrossRef Link).

[4] Y. Amichai-Hamburger and G. Vinitzky, "Social network use and personality," Computers in Human Behavior, vol.26, No.6, pp.1289-1295, November, 2010. Article (CrossRef Link).

[5] G. Mulgan, "Government, knowledge and the business of policy making: the potential and limits of evidence-based policy," Evidence \& Policy, Journal of Research, Debate and Practice, vol.1, no.2, pp.215-216, May, 2005. Article (CrossRef Link).

[6] D. Ariely, A. Bracha and S. Meier, "Doing good or doing well? Image motivation and monetary incentives in behaving prosocially,” American Economic Review, vol.99, no.1, pp.544-555, March, 2009. Article (CrossRef Link).

[7] S. Aral, E. Brynjolfsson and M. Van Alstyne, "Productivity effects of information diffusion in networks". Available at SSRN 987499, 2007. Article (CrossRef Link).

[8] J. Fontdevila, M. P. Opazo and H. C. White, "Order at the Edge of Chaos: Meanings from Netdom Switchings across Functional Systems,” Sociological Theory, vol.29, no.3, pp.178-198, September, 2011. Article (CrossRef Link).

[9] F. Godart and H. White, "Switchings under uncertainty: The coming and becoming of meanings," Poetics, vol.38, no.6, pp.567-586, December, 2010. Article (CrossRef Link).

[10] H. White, J. Fuhse, M. Thiemann and L. Buchholz, "Networks and Meaning: Styles and Switchings,” Soziale Systeme, vo.13, no.1-2, pp.543-555, May, 2007. Article (CrossRef Link).

[11] S. Faraj, S. Jarvenpaa and A. Majchrzak, "Knowledge collaboration in online communities," Organization Science, vol.22, no.5, pp.1224-1239, February, 2011. Article (CrossRef Link).

[12] J. Füller, K. Hutter and R. Faullant, "Why co-creation experience matters? Creative experience and its impact on the quantity and quality of creative contributions," R\&D Management, vol.41, no.3, pp.259-273, May, 2011. Article (CrossRef Link).

[13] R. Watson, M. Boudreau, M. C., S. Li and J. Levis, "Telematics at UPS: En Route to Energy Informatics,” MIS Quarterly Executive, vol.9, no.1, pp.1-11, 2010. Article (CrossRef Link).

[14] A. Susarla, J. Oh and Y. Tan, "Social networks and the diffusion of user-generated content: Evidence from You-tube," Information Systems Research, vol.23, no.1, pp.23-41, April, 2011. Article (CrossRef Link).

[15] L. Salamon, and S. Sokolowski, "Institutional roots of volunteering” pp. 71-90. Springer US, 2003. Article (CrossRef Link).

[16] J. Kim, S. Sterman, A. Cohen and M. Bernstein, Mechanical novel: Crowdsourcing complex work through reflection and revision. In Design Thinking Research, pp.79-104, Springer, Cham, 2018. Article (CrossRef Link).

[17] H. White, Identity and Control: How social formation emerge, Princeton university press, 
Princeton, USA, NJ, 2008. Article (CrossRef Link).

[18] B. Wellman. "Networks in the global village: Life in contemporary communities," Routledge, USA, NY, 2018. Article (CrossRef Link).

[19] C. Okoli, and W. Oh, "Investigating recognition based performance in an open content community: A social capital perspective," Information and Management, vol.44, no.3, pp.240-252, April, 2007. Article (CrossRef Link).

[20] R. Swedberg, “Joseph A. Schumpeter: his life and work,” John Wiley \& Sons, 2013. Article (CrossRef Link).

[21] D. Centola and M. Macy, "Complex contagions and the weakness of long ties," American Journal of Sociology, vol.13, No.3, pp.702-734, November, 2007. Article (CrossRef Link).

[22] A. Mische and H. White, "Between conversation and situation: Public switching dynamics across network domains," Social Research, vol.65, no.3, pp.695-724, Fall, 1998. Article (CrossRef Link).

[23] J. Padgett and C. Ansell, "Robust Action and the Rise of the Medici," 1400-1434. American Journal of Sociology, vol.98, no.6, pp.1259-1319, 1993. Article (CrossRef Link).

[24] J. Kim and H. Marakand, "Social network analysis," International Journal of Information Management: The Journal for Information Professionals, vol.38, no.1, pp.86-96, February, 2018. Article (CrossRef Link).

[25] T. Kim and M. Rhee, "Exploration and exploitation: Internal variety and environmental dynamism,” Strategic Organization, vol.7, no.1, pp.11-41, February, 2009. Article (CrossRef Link).

[26] X, Li, W. Shi and B. Zhu, "The face of internet recruitment: Evaluating the labor markets of online crowdsourcing platforms in China," Research \& Politics, vol.5, no.1, pp1-8, February, 2018. Article (CrossRef Link).

[27] J. Sidhu, H. Volberda and H. Commandeur, "Exploring exploration orientation and its determinants: some empirical evidence," Journal of Management Studies, vol.41, no.6, pp.913-932, August, 2004. Article (CrossRef Link).

[28] R. Cyert and J. March, A behavioral theory of the firm. Englewood Cliffs, NJ, 2, 1963.

[29] L. Dabbish and. R. Kraut, "Controlling interruptions: awareness displays and social motivation for coordination," in Proc. of Proceedings of the 2004 ACM conference on Computer supported cooperative work, pp.182-191, November 6-10, 2004. Article (CrossRef Link).

[30] A. Animesh, A. Pinsonneault, S. Yang and W. Oh, "An Odyssey into virtual worlds: exploring the impacts of technological and spatial environments on intention to purchase virtual products," MIS Quarterly, vol.35, no.3, pp.789-810, September, 2011. Article (CrossRef Link).

[31] P. Podsakoff, S. MacKenzie, J. Lee and N.Podsakoff, "Common method biases in behavioral research: a critical review of the literature and recommended remedies," Journal of applied psychology, vol.88, no.5, pp.879-881, 2003. Article (CrossRef Link).

[32] W. Chin, "The partial least square approach to structural equation modeling," G. A. Marcoulides (ed.), Modern Methods for Business Research, pp. 295-336, Lawrence Erlbaum Associates, Mahwah, NJ, 1998. Article (CrossRef Link).

[33] R. Bagozzi, Y. Yi and L. Phillips, "Assessing construct validity in organizational research," Administrative Science Quarterly, vol.36, no.3, pp.421-458, September, 1991. Article (CrossRef Link).

[34] M. Chung, M. and J. Kim, "The internet information and technology research directions based on the fourth industrial revolution," KSII Transactions on Internet \& Information Systems, vol.10, no.3, pp.1311-1320, March, 2016. Article (CrossRef Link).

[35] K. Chang and J. Chen. "A survey of trust management in WSNs, internet of things and future internet," KSII Transactions on Internet \& Information Systems, vol.6, no.1, pp.5-23, January, 2012. Article (CrossRef Link). 


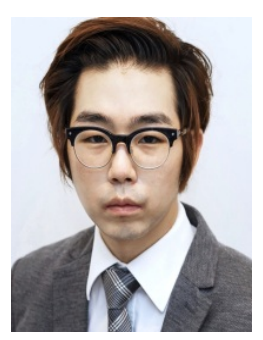

Jemin Justin Lee received his B.A. degree from Glion Institute of Higher Education, Switzerland, in 2013, and a Ph.D. degree in Electrical and Computer Engineering from Yonsei University, Republic of Korea. Since 2018, he has been a Research Professor of the Communications and Networking Laboratory (CNL) at Yonsei University. His research interests are on Cloud Computing, Internet of Things (IoT), and Big Data.

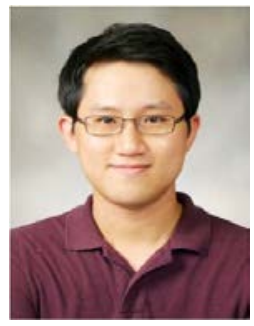

Youngjoon Cheon is a director of corporate relations at Youngpoong corporation. He received his Ph.D. in Technology policy in Yonsei university, south Korea, in 2016. His research interests are in the field of 'ICT policy', 'Agriculture and ICT', 'Social Innovation and Economy'.

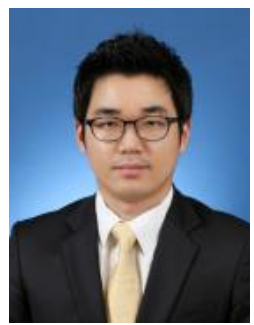

Sangyun Han is an assistant professor at the Department of International Commerce at the Daejeon University in Republic of Korea. He received his doctorate in Business Administration from Yonsei University. His research focuses on management of technology innovation with a specific interest in external knowledge internalization, diffusion of innovative technology.

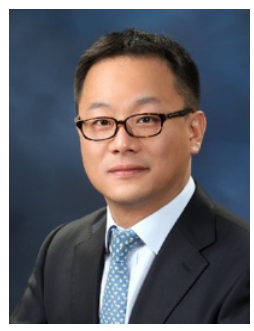

Kyu Tae Kwak is an assistant professor at the Department of Global Culture Industry; College of Global Business in Soonchunhyang University since 2017. He received his Ph.D. in Business Administration from Yonsei University, South Korea, in 2014. His research interests are in the field of 'Creativity and Innovation', 'Media Management', 'Content Business'. 